\title{
Sentinel lymph node biopsy. A prospective medical advance to substitute the pelvic lymphadenectomyin early cervical cancer. A reappraisal.
}

\author{
Anastasios Pandraklakis ${ }^{1}$, Nikolaos Thomakos ${ }^{1}$, loannis Thanopoulos ${ }^{1}$, Dimitrios Vlachos ${ }^{1}$, \\ Maria D. Oikonomou' ${ }^{2}$, Dimitrios Chaidopoulos ${ }^{1}$, Alexandros Rodolakis ${ }^{1}$ \\ ${ }^{1} 1$ st Department of Obstetrics and Gynecology, "Alexandra" Maternity Hospital, National and Kapodistrian \\ University of Athens, Athens, Greece \\ 23rd Department of Obstetrics and Gynecology,"Attikon" Hospital, National and Kapodistrian University of \\ Athens, Athens, Greece
}

Correspondence

Correspondence should be addressed to Anastasios Pandraklakis; tasospandraklakis@hotmail.com

Key words: Sentinel lymph node, SLN, cervival cancer, ultrastaging, low volume disease, LVD, micromestastasis, isolated tumor cells

\section{Introduction}

Cervical cancer is the fourth most common malignancy in the female population and was the ninth cause of deaths in the female population in $2016^{1}$. According to the American Cancer Society, 13240 new cases of cervical cancer will be diagnosed in the USA in the year 2018, while 4170 women will die from the disease ${ }^{2}$. Unfortunately, $40 \%$ of them concern women younger than 45 years old in their reproductive age ${ }^{3}$.

Traditionally, the therapeutic strategy for early stage cervical cancer (IA1-IB1) includes radical hysterectomy and bilateral pelvic lymphadenectomy (Wertheim's procedure)with excellent oncologic outcomes ${ }^{4}$. Despitethough the excellent oncologic results of the procedure, sterilization, sexual urological dysfunction and lymphedemaare notable disadvantages of the surgical management ${ }^{5}$.
Cervical cancer staging, according to FIGO, unlike other gynecologic neoplasms is not a surgical process.Although not included in the FIGO clinical staging, the pelvic and para-aortic lymph node status is an important prognostic factor in cervical cancerstaging because the survival rates of patients with metastases to the nodes are significantly lower than these of patients without metastases to the nodes ${ }^{6,7}$. Therefore, an accurate staging and detection of lymph node(LN)metastases is essential in order to ensure appropriate treatment planning and prediction of prognosis for cervical cancer patients ${ }^{8}$.

A number of studies has evaluated the diagnostic performance of non-invasive modalities, such as CT, MRI and positron emission tomography (PET)-2-[18 F] fluoro2-deoxy-D-glucose (FDG) in detecting LN metastasis in cervical cancer patients. MRI and CT 
have been conventionally and widely used to detect LN metastases based on measurements like node size and morphologic information, with low sensitivity and specificity rates though. PET and PET/CT offer combined benefits of anatomic and functional imaging and have been used to localize areas of increased FDG uptake with improved anatomic specificity ${ }^{9}$. However, controversy still exists in relation to the application of PET/CT in clinical practice, mainly because of its high $\operatorname{cost}^{10}$. Furthermore, frozen section biopsy of LNs is an intraoperative module which is characterized by low accuracy rates in detection of metastases with high false negative rates for stages IA2 - IB1 $(4.2 \%)^{11}$.

\section{Sentinel Lymph Node (SLN) phylosophy}

Sentinel Lymph Node(SLN) is the first lymph node or group of nodes draining an anatomic area. In case of an established cancerous dissemination it is postulated that the sentinel node is the target organ primarily reached by metastasizing cancer cells from the tumor. Breast, penile, and skin cancer treatments have established the SLN procedure while negative SLN tests have helped avoid full lymph node dissection.Data from several studies of SLN biopsy in early cervical cancer are also satisfactory. In most studies the detection rates fluctuate between 95 and 100 per cent, false negative rates remain between 0 and 8 per cent and their negative predictive value is found between 97 and 100 per cent. Thus, in order to avoid complications associated with lymphadenectomy,SLN biopsy has been introduced in the surgical managementof cervical cancer ${ }^{12}$.

Anatomically, the most common drainage is the lymphatics that come out through the broad ligament. These lymphatics cross over the umbilical ligament as they end up in classic mapping areas such as the iliac or the obturator area and are most commonly found medially to the external iliac vessels and ventrally to the hypogastric superior part of the obturator ${ }^{13,14}$.

\section{Clinical Utility}

Lymph node metastasis rates in women with early cervical cancer are foundbetween $15-20 \%{ }^{15}$. Therefore, according to the traditional strategies, $80 \%$ of women will not benefit from pelvic lymphadenectomy and its associated complications(lymphatic cysts, lymphedema, urologic disorders and sexual dysfunction). Thus, using the SLN technique, these women with negative SLN could omit full pelvic lymphadenectomy ${ }^{5}$.

Frequently, patients demonstrate pelvic recurrency despite the fact that lymphadenectomy has been performed. This is due to lymph node involvement in atypical locations, in approximately $10 \%$ of women, (presacral, lower paraortic, common illiac, medial part of lateral parametrium)which was not excised during pelvic lymphadenectomy (rob et al gyn oncol 2005). Rob et al. revealedthat positive sentinel node rates in these atypical locations are about $15 \%{ }^{16}$.

As already mentioned, the currentmanagement of women with early cervical cancer is radical hysterectomy(RH) with bilateral pelvic lymphadenectomy, while in cases that the histologic diagnosis reveals positive lymph nodes, patients will be referred for adjuvant radiotherapy(RT) ${ }^{17}$. When using the SLN procedure, if a woman has a positive SLN, the hysterectomy is abandoned and the patient is referred for primary $\mathrm{RT}^{18}$. If the SLN is negative then the patient can undergo $\mathrm{RH}$ or a more conservative fertility sparing treatment ${ }^{19}$.

The most attractive idea is to use the SLN procedure for triaging patients in order to prevent women with positive lymph nodes from radical operations ${ }^{20}$. Furthermore, these women can be referred for another treatment modality (adjuvant radiotherapy), while omitting all the negative consequences ${ }^{20}$. Attention should be paid to patients with false negative 
SLN because an "overtreating" scenario with a radical surgery is possible ${ }^{21}$.

On the other handthe SLN procedure can be used to select patients who are candidates for less radical procedures when the SLNs are negative. In this case a false negative SLN can lead to "undertreatment" of patients because of the high false negative rates of the technique ${ }^{21}$.

Moreover, even if several studies have been conducted, the procedure is not yet established because of the severe false negative rates in patients with early stage cervical tumors. This fact leads in a demised trustworthiness of the SLN procedure and therefore in insufficient metastasis detection. Consequently, the challenge in protocol development is to detect micrometastases that can be missed ${ }^{18}$.

Low volume disease (LVD) is a new terminology that has been introduced in gynecological cancers. LVD concerns tumors smaller than $2 \mathrm{~mm}^{22}$. Micrometastasis is a low volume metastasis measuring $0,2-2 \mathrm{~mm}$ in the form of microscopic clusters and single cells while Isolated tumor cells (ITC) concern metastasis measuring lower than $0.2 \mathrm{~mm}^{22}$.

Studies have shown that the survival rate in patients with micrometastasis seems to be equal to patients carrying macrometastasis ${ }^{23}$. Furthermore, isolated tumor cells do not seem to affect survival rates $^{23}$. Therefore, the "ultrastaging protocol"has come to ameliorate the intraoperative histology procedures as it focuses in the detection of the metastases that could be potentially missed ${ }^{22}$.

When an SLN is sent for frozen section, a micrometastasis can be potentially included on the half node that will not be cut for examination. Consequently, the improvement of pathologic intraoperative processing is the activation of the Ultrastaging protocol. Ultrastaging in negative SLNs is a promising technique that can detect low volume metastasis. This protocol is activated by pathologists in cases of negative SLNs. With this process, further sectioning of the lymph node will be able to capture a $2 \mathrm{~mm}$ metastasis that may have been missed on the first cut $^{22,24}$. This technique consists of multiple serial sectioning of the negative SLNs and examination of them using immunohistochemistry methodsgiving the possibility to detect LVD which could be missed in the ordinary frozen section procedure. This way, even the $10 \%$ of negative SLN with micrometastasis can be diagnosed and moreover, the treatment strategy for these women who would not be referred to adjuvant RT will change ${ }^{23}$.

Results from several studies have shown that the detection rate of SLN in cervical cancer is close to $99 \%$ and that the bilateral detection rate is $85 \%$, especially with the use of Indocyanine green technique $^{25}$.Sensitivity in bilateral detection of SLN reaches up to $97 \%$ and false negative rates to $1,3 \%{ }^{23}$. However, Zarganis et al. revealed higher false negative rates in larger tumors, especially in stages IB2 and IIA $^{26}$. Tax $\mathrm{C}$ et al. showed that the residual risk on occult metastasis in early cervical cancer patients with negative SLNs after ultrastaging is $0.08 \%{ }^{21}$.

\section{Conclusion}

In conclusion, the question at hand is whether the medical society is ready to replace systematic pelvic lymphadenectomy in early cervical cancer forthe SLN biopsy only. This matter is under investigation in the Senticol III and the Sentix randomized prospective trials and their results are expectedin order to establish detailed guidelines for the SLN biopsy in cervical cancer.

\section{References}

1. Department of Health Hong Kong. Statistics of Cervical Cancer. 2016;(March):1-3.

2. Siegel RL, Miller KD, Jemal A. Cancer statistics, 2018. CA Cancer J Clin. 2018;68(1):7-30. doi:10.3322/caac.21442.

3. Noone A, Howlader N, Krapcho M, et al. SEER 
Cancer Statistics Review, 1975-2015.; 2017.

4. American Cancer Society. Cancer Facts \& Figures 2018.; 2018. doi:10.1097/01.NNR.0000289 503.22414.79.

5. Mathevet P, Lecuru F, Magaud L, Bouttitie F. Sentinel lymph node biopsy for early cervical cancer: Results of a randomized prospective, multicenter study (Senticol 2) comparing adding pelvic lymph node dissection vs sentinel node biopsy only. Gynecol Oncol. 2017;145:2-3. doi:10.1016 /j.ygyno.2017.03.029.

6. Chung HH, Park N-H, Kim JW, Song Y-S, Chung J$\mathrm{K}$, Kang S-B. Role of integrated PET-CT in pelvic lymph node staging of cervical cancer before radical hysterectomy. Gynecol Obstet Invest. 2009;67(1). doi:10.1159/000162101.

7. Reinhardt MJ, Ehritt-Braun C, Vogelgesang D, et al. Metastatic Lymph Nodes in Patients with Cervical Cancer: Detection with MR Imaging and FDG PET. Radiology. 2001;218(3):776-782..

8. Grueneisen J, Schaarschmidt BM, Heubner M, et al. Integrated PET/MRI for whole-body staging of patients with primary cervical cancer: preliminary results. Eur J Nucl Med Mol Imaging. 2015;42(12):1814-1824. doi:10.1007/s00259015-3131-5.

9. Liu B, Gao S, Li S. A comprehensive comparison of CT, MRI, positron emission tomography or positron emission tomography/CT, and diffusion weighted imaging-MRI for detecting the lymph nodes metastases in patients with cervical cancer: A meta-analysis based on 67 studies. Gynecol Obstet Invest. 2017;82(3):209-222. doi:10.1159 /000456006.

10. Auguste P, Barton P, Meads C, et al. Evaluating PET-CT in routine surveillance and follow-up after treatment for cervical cancer: A cost-effectiveness analysis. BJOG An Int J Obstet Gynaecol. 2014;121(4):464-476. doi:10.1111/1471-0528. 12460.
11. Panici PB, Maggioni A, Hacker N, et al. Systematic aortic and pelvic lymphadenectomy versus resection of bulky nodes only in optimally debulked advanced ovarian cancer: A randomized clinical trial. J Natl Cancer Inst. 2005;97(8):560566. doi:10.1093/jnci/dji102.

12. R.H.M. V, R. P, P.J. VD, C.W. B, M.R. B, P. K. Sentinel node detection in cervical cancer. Obstet Gynecol. 2000;96(1):135-138. doi:10.1016/S0029-7844 (00)00831-0.

13. Kurman edited by RJ. Blaustein's Pathology of the Female Genital Tract (4th Ed.).; 1994.

14. Singer A. The Vascular, Neural and Lymphatic Anatomy of the Cervix". The Cervix (2nd ed.). In: The Cervix. ; 2005.

15. Delgado G, Bundy BN, Fowler WC, et al. A prospective surgical pathological study of stage I squamous carcinoma of the cervix: A Gynecologic Oncology Group study. Gynecol Oncol. 1989; 35(3):314-320. doi:10.1016/0090-8258 (89)90 070-X.

16. Rob L, Strnad P, Robova H, et al. Study of lymphatic mapping and sentinel node identification in early stage cervical cancer. Gynecol Oncol. 2005;98(2):281-288. doi:10.1016/ j.ygyno.2005. 04.016

17. Lennox GK, Covens A. Can sentinel lymph node biopsy replace pelvic lymphadenectomy for early cervical cancer? Gynecol Oncol. 2017;144(1):1620. doi:10.1016/j.ygyno.2016.08.337.

18. Lécuru F, Mathevet P, Querleu D, et al. Bilateral negative sentinel nodes accurately predict absence of lymph node metastasis in early cervical cancer: Results of the SENTICOL study. J Clin Oncol. 2011;29(13):1686-1691. doi:10.1200/ JC0.2010.32.0432.

19. Du X, Sheng X, Jiang T, et al. Sentinel lymph node biopsy as guidance for radical trachelectomy in young patients with early stage cervical cancer. BMC Cancer. 2011;11(1):157. doi:10.1186/ 
1471-2407-11-157.

20. Cibula D, Kuzel D, Sláma J, et al. Sentinel node (SLN) biopsy in the management of locally advanced cervical cancer. Gynecol Oncol. 2009;115(1):46-50. doi:10.1016/ j.ygyno.2009. 06.017 .

21. Tax C, Rovers MM, De Graaf C, Zusterzeel PLM, Bekkers RLM. The sentinel node procedure in early stage cervical cancer, taking the next step; A diagnostic review. Gynecol Oncol. 2015; 139(3):559-567. doi:10.1016/j.ygyno.2015. 09.076.

22. Euscher ED, Malpica A, Atkinson EN, Levenback CF, Frumovitz M, Deavers MT. Ultrastaging improves detection of metastases in sentinel lymph nodes of uterine cervix squamous cell carcinoma. Am J Surg Pathol. 2008;32(9). doi:10.1097/ PAS.0b013e31816ecfe4.

23. Cibula D, Abu-Rustum NR, Dusek L, et al. Prognostic significance of low volume sentinel lymph node disease in early-stage cervical cancer. Gynecol Oncol. 2012;124(3):496-501. doi:10.1016/ j.ygyno.2011.11.037.
24. Sams RN, Euscher ED, Malpica A. Sentinel lymph nodes in cervical cancer: A first cut analysis. Lab Investig. 2014;94:511A-512A.

25. Kim J, Kim D, Suh D, Kim J, Kim Y, Kim Y. The efficacy of sentinel lymph node mapping with indocyanine green in cervical cancer. 2018:1-7.

26. Zarganis P, Kondi-Pafiti A, Arapantoni-Dadioti P, et al. The sentinel node in cervical cancer patients: role of tumor size and invasion of lymphatic vascular space. In Vivo. 2009;23(3): 469-473.
Received 1-5-2018

Revised 22-5-2018

Accepted 20-6-2018 